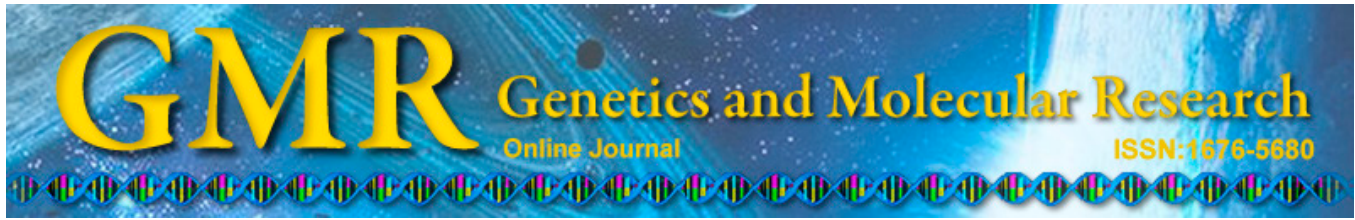

\title{
Role of Nrf2 signal pathway in rats with deep hypothermia ischemia/reperfusion injury undergoing remote postconditioning
}

\author{
Z.Y. Wu*, Z.W. Wang*, R. Hu, Z. Zhou, L.C. Li and Z.F. Mao \\ Department of Cardiovascular Surgery, \\ Renmin Hospital of Wuhan University, Wuhan, China \\ *These authors contributed equally to this study. \\ Corresponding author: Z.Y. Wu \\ E-mail: zhiyongwu889@sina.com
}

Genet. Mol. Res. 14 (1): $492-499$ (2015)

Received November 11, 2013

Accepted June 6, 2014

Published January 26, 2015

DOI http://dx.doi.org/10.4238/2015.January.26.2

\begin{abstract}
We investigated the protective effects of remote postconditioning (RPC) in the lungs of rats with deep hypothermia ischemia/reperfusion ( $\mathrm{I} / \mathrm{R})$ injury and the role of nuclear factor E2related factor $2(\mathrm{Nrf2})$ signaling in this process. Forty-nine rats were randomly divided into a sham control group, deep hypothermia I/R group, RPC group, I/R+all-trans retinoic acid (ATRA) group, I/ $\mathrm{R}+\mathrm{RPC}+\mathrm{ATRA}$ group, I/R+tert-butylhydroquinone (tBHQ) group, and $\mathrm{I} / \mathrm{R}+\mathrm{RPC}+\mathrm{tBHQ}$ group. Real-time polymerase chain reaction and Western blot analysis were used to examine Nrf2 mRNA and protein expression, respectively. Compared with the sham control group, Nrf2 expression, malondialdehyde (MDA) content, and the wet/dry weight (W/D) ratio were significantly increased in the I/R group, while superoxide dismutase (SOD) activity was significantly decreased. Pulmonary Nrf2 expression and SOD activity was significantly increased, and MDA content and the W/D ratio were significantly decreased in the RPC group compared with the I/R group. Compared with the I/R group, MDA and W/D ratio significantly decreased and SOD activity remarkably increased in $\mathrm{I} / \mathrm{R}+\mathrm{tBHQ}$ group. After ATRA
\end{abstract}


intervention in the I/R+ATRA group, MDA content and W/D ratio increased and SOD activity decreased compared to the I/R group. MDA content and W/D ratio in the RPC + tBHQ group significantly decreased and SOD activity increased compared with in the RPC group $(\mathrm{P}<0.01)$. In the RPC+ATRA group, MDA content and W/D ratio decreased while SOD activity increased compared with the RPC group $(\mathrm{P}<0.01)$. RPC alleviated deep hypothermia I/R injury; the Nrf2 signaling pathway may be involved in the protective effects induced by RPC.

Key words: All-trans retinoic acid; Nrf2 signaling pathway; Deep hypothermia ischemia-reperfusion injury; tBHQ;

Remote postconditioning

\section{INTRODUCTION}

Remote postconditioning (RPC), an endogenous mechanism that protects organs, was recently proposed after the identification of ischemic preconditioning and ischemic postconditioning. Ischemia-reperfusion (I/R) injury of the ischemic organs can be diminished when brief ischemia-reperfusion is applied to a distant organ such as the kidney and skeletal muscle (Xin et al., 2010). A previous study showed that the possible mechanism of RPC involves the activation of protein kinase, phosphatidylinositol-3-kinase/protein kinase B, extracellular signal-regulated kinase, heme oxygenase-1, adenosine receptors, ATP-sensitive potassium channel, and inhibition of apoptosis (Ke et al., 2010; Tang et al., 2010; Tsubota et al., 2010). The protein kinase $\mathrm{C}$, phosphatidylinositol-3-kinase (PI3K)/protein kinase $\mathrm{B}$, and extracellular signal-regulated kinase pathways were found to be involved in activation of the nuclear factor E2-related factor 2 (Nrf2) pathway, a master regulator of the cellular redox state (Hochmuth et al., 2011). Heme oxygenase-1, produced after the activation of Nrf2 as one of the phase 2 detoxification enzymes, is also related to the RPC mechanism (Osburn and Kensler, 2008). Therefore, we examined whether deep hypothermia I/R injury of rat lungs could be prevented via the Nrf2 pathway as the common downstream pathway of protein kinase $\mathrm{C}, \mathrm{PI} 3 \mathrm{~K} /$ protein kinase B, and extracellular signal-regulated kinase.

In this study, all-trans retinoic acid (ATRA) and tert-butyl hydroquinone (tBHQ) were used as an antagonist and agonist of the Nrf2 pathway, respectively. We investigated the potential role of the Nrf2 signal pathway in the lungs of rats with deep hypothermia I/R injury.

\section{MATERIAL AND METHODS}

\section{Animals}

Specific pathogen-free Wistar rats used in this study (200-250 g, N = 49) were obtained from Hubei Research Center of Experimental Animals. The animals were housed at a humidity of $50-80 \%$ and temperature of $24^{\circ}-27^{\circ} \mathrm{C}$. The use of animals in this study was approved by the Ethics and Research Committee of Renmin Hospital of Wuhan University. All animal procedures were in accordance with the Principles of Laboratory Animal Care formulated by the National Society for Medical Research and the Guide for the Care and Use of Laboratory Animals published by the National Institutes of Health (NIH publication 85-23, revised 1996). 


\section{Experimental design}

Forty-nine rats were randomly divided into: a) a sham control group $(\mathrm{N}=7)$, which were subjected to sham operation; b) a deep hypothermia I/R injury group $(\mathrm{N}=7)$, which underwent occlusion of the bronchial arteries, left inferior pulmonary vein, and left inferior pulmonary artery; c) an RPC group $(\mathrm{N}=7)$, which were subjected to $\mathrm{I} / \mathrm{R}$ injury and treated with RPC; d) an I/R+ATRA group ( $\mathrm{N}=7)$, which were subjected to $I / R$ injury and treated with ATRA ( $20 \mathrm{mg} \cdot \mathrm{kg}^{-1} \cdot \mathrm{day}^{-1}$ for 2 days via intraperitoneal injection; Sigma-Aldrich, St. Louis, $\mathrm{MO}$, USA); e) an I/R+RPC+ATRA group $(\mathrm{N}=7)$, which were subjected to I/R injury and treated with RPC and ATRA, respectively; f) an I/R+tBHQ group $(\mathrm{N}=7)$, which were subjected to I/R injury and treated with tBHQ $\left(20 \mathrm{mg} \cdot \mathrm{kg}^{-1} \cdot \mathrm{day}^{-1}\right.$ for 2 days via intraperitoneal injection; Sigma-Aldrich); g) an $\mathrm{I} / \mathrm{R}+\mathrm{RPC}+\mathrm{tBHQ}$ group $(\mathrm{N}=7)$, which were subjected to $\mathrm{I} / \mathrm{R}$ injury and treated with RPC and tBHQ.

Wistar rats in the I/R group were anesthetized via intraperitoneal injection of $0.3 \%$ pentobarbital sodium ( $40 \mathrm{mg} / \mathrm{kg}$ body weight). The animal's neck was shaved using a hair clipper. Next, a vertical incision (approximately $1 \mathrm{~cm}$ in length) was made. After the laryngeal muscle was separated to expose the trachea, an intubation tube was inserted through the oral cavity. The tube was connected to a respirator with a respiratory frequency of $80-90 / \mathrm{min}$. The respiratory exchange ratio was set at 1:2-1:3 with a working pressure of 2-4 $\mathrm{kPa}$. Thoracotomy was carried out at the 4th intercostal space. Occlusion of the bronchial arteries, left inferior pulmonary vein, and left inferior pulmonary artery was performed using a forceps clip after heparinization $(1.5 \mathrm{mg} / \mathrm{kg})$ under profound hypothermia conditions. After $30 \mathrm{~min}$, the clips were removed and rewarming of the rats was conducted using an infrared lamp. For the sham control group, only thoracotomy was performed with no occlusion of the left lung.

\section{Measurement of malondialdehyde (MDA) and superoxide dismutase (SOD) activity}

To measure the level of MDA, $0.1 \mathrm{~g}$ tissues were incubated at $95^{\circ} \mathrm{C}$ for $40 \mathrm{~min}$ after homogenization. The samples were cooled to room temperature and centrifuged for $10 \mathrm{~min}$ at $980 \mathrm{~g}$. MDA level was determined using the method described by Satoh (1978) using the thiobarbituric acid reaction; the products were separated by high-performance liquid chromatography. To measure SOD activity, the tissue was incubated for $10 \mathrm{~min}$ at room temperature after homogenization. Ultraviolet detection of SOD activity and MDA was performed at 532 and $550 \mathrm{~nm}$, respectively.

\section{Measurement of lung water content}

After sacrificing the animals, pulmonary tissues were immediately removed from the inferior lobe of the left lung. Coronal slices with a depth of $4 \mathrm{~mm}$ were prepared. The wet weight (W) of each slice was determined using an electronic analytical balance. The slices were dried in an oven at $100^{\circ} \mathrm{C}$ for $24 \mathrm{~h}$ until the dry weight (D) could be obtained. Water content $(\%)$ of the pulmonary tissues was calculated as $[(\mathrm{W}-\mathrm{D}) / \mathrm{W}] \mathrm{x} 100 \%$.

\section{Reverse transcription-polymerase chain reaction (PCR) analysis for Nrf2 mRNA}

Total mRNA was extracted using TRIzol reagent according to manufacturer in- 
structions (Invitrogen, Carlsbad, CA, USA). cDNA was synthesized using M-MLV reverse transcriptase (Invitrogen) according to manufacturer instructions. Real-time PCR was performed using SYBR Green Supermix on a 7500 Fast RT-PCR system (Applied Biosystems Inc., Foster City, CA, USA). The mRNA level was normalized by $\beta$-actin. The primers used for $N r f 2$ were 5'-CCCAGCACATCCAGACAGAC-3' and 5'-TATCCAGGGCAAGCGA CTC-3'. The cDNA of $N r f 2$ was amplified by PCR $\left(30\right.$ cycles: $94^{\circ} \mathrm{C}, 25 \mathrm{~s} ; 60.5^{\circ} \mathrm{C}, 25 \mathrm{~s}$; and $72^{\circ} \mathrm{C}, 25 \mathrm{~s}$ ) using $2 \mathrm{~mL}$ cDNA (10X dilution) according to manufacturer instructions. The primers for $\beta$-actin were 5'-CCTCATGAAGATCCTGACCG-3' and 5'-ACCGCTCATTGCCG ATAGTG-3'. The amplification results for real-time PCR were calculated using the $2^{(-\Delta \Delta C t)}$ method, as previously described (Beillard et al., 2003).

\section{Western blotting analysis of Nrf2 expression in pulmonary tissues}

Western blotting analysis was performed as previously described (Unver et al., 2001). Briefly, lung tissue was lysed using RIPA lysis buffer in an ice bath for $1 \mathrm{~h}$. The sample was centrifuged at $14,000 \mathrm{rpm}$ at $4^{\circ} \mathrm{C}$. The proteins were separated by electrophoresis on a $12 \%$ sodium dodecyl sulfate-polyacrylamide gel and transferred to a Hybond-P polyvinylidene fluoride membrane. The membrane was blocked using 5\% nonfat milk and incubated with a rabbit anti-rat Nrf2 antibody (1:500) overnight at $4{ }^{\circ} \mathrm{C}$, followed by incubation with horseradish peroxidase-conjugated goat anti-rabbit secondary antibody (1:5000) for $2 \mathrm{~h}$ at room temperature. After washing with phosphate-buffered saline, the bound primary antibody was visualized using the Odyssey ${ }^{\mathbb{B}}$ Infrared Imaging System (Licor Bioscience Inc., Lincoln, NE, USA) and exposed to film. The same membrane was probed for $\beta$-actin as a loading control. The relative density of Nrf2 to $\beta$-actin was analyzed using the LabWorks 415 software (Upland, CA, USA).

\section{Histopathological examination}

Sections were embedded with $10 \%$ paraformaldehyde and cut to a thickness of $4 \mathrm{~mm}$. Sections were stained with routine hematoxylin and eosin. The histological structures of the sections were observed using an Olympus IX51 microscope (Olympus Inc., Tokyo, Japan) at a magnification of $200 \mathrm{X}$.

\section{Statistical analysis}

All data are reported as means \pm standard deviation. The SPSS 13.0 software was used for data analysis (SPSS, Inc., Chicago, IL, USA). Analysis of variance was performed for inter-group comparisons. $\mathrm{P}<0.05$ was considered to be statistically significant.

\section{RESULTS}

\section{Determination of MDA content, SOD activity, and W/D ratio in pulmonary tissues}

A significant increase in MDA content and W/D ratio was observed in the $\mathrm{I} / \mathrm{R}$ group compared with the sham control group $(\mathrm{P}<0.01$; Table 1); in addition, a remarkable decrease in SOD activity was observed in the I/R group compared with the sham control group $(\mathrm{P}<$ 0.01). Compared with the I/R group, a significant decrease in MDA content and W/D ratio 
was observed in the RPC group. SOD activity showed a remarkable increase in the RPC group compared with the I/R group $(\mathrm{P}<0.01)$. Compared with the $\mathrm{I} / \mathrm{R}$ group, a significant decrease in MDA content and W/D ratio was observed in the $\mathrm{I} / \mathrm{R}+\mathrm{tBHQ}$ group $(\mathrm{P}<0.01)$, while SOD remarkably increased in the $\mathrm{I} / \mathrm{R}+\mathrm{tBHQ}$ group $(\mathrm{P}<0.01)$. After ATRA intervention in the $\mathrm{I} /$ $\mathrm{R}+$ ATRA group, MDA content and the W/D ratio were remarkably increased compared with the $\mathrm{I} / \mathrm{R}$ group $(\mathrm{P}<0.01)$, while SOD activity remarkably decreased compared with the $\mathrm{I} / \mathrm{R}$ group $(\mathrm{P}<0.01)$. MDA content and the $\mathrm{W} / \mathrm{D}$ ratio in the $\mathrm{RPC}+\mathrm{tBHQ}$ group were significantly decreased compared with the RPC group $(\mathrm{P}<0.01)$, while SOD activity was increased compared with the RPC group $(\mathrm{P}<0.01)$. MDA content and the W/D ratio were significantly decreased in the RPC+ATRA group compared with the RPC group $(\mathrm{P}<0.01)$, while SOD activity was significantly increased compared with the RPC group $(\mathrm{P}<0.01)$.

\begin{tabular}{|c|c|c|c|}
\hline Group & MDA (nmol/mg protein) & SOD (U/mg protein) & $\mathrm{W} / \mathrm{D}$ \\
\hline Sham & $1.34 \pm 0.31$ & $48.19 \pm 4.24$ & $3.25 \pm 0.37$ \\
\hline $\mathrm{I} / \mathrm{R}$ & $5.18 \pm 0.59^{\#}$ & $19.83 \pm 1.41^{\#}$ & $5.27 \pm 0.92^{\#}$ \\
\hline $\mathrm{RPC}$ & $2.77 \pm 0.26^{\bullet}$ & $31.74 \pm 2.83^{\bullet}$ & $3.98 \pm 0.54^{\bullet}$ \\
\hline I/R+ATRA & $6.10 \pm 0.73^{\triangle}$ & $10.85 \pm 1.76^{\triangle}$ & $6.12 \pm 0.51^{\triangle}$ \\
\hline RPC+ATRA & $4.84 \pm 0.49^{£}$ & $20.70 \pm 3.17^{\&}$ & $4.57 \pm 0.66^{\&}$ \\
\hline $\mathrm{I} / \mathrm{R}+\mathrm{tBHQ}$ & $3.96 \pm 0.41^{\boldsymbol{V}}$ & $29.64 \pm 2.89^{\mathbf{V}}$ & $4.97 \pm 0.51^{\mathbf{}}$ \\
\hline $\mathrm{RPC}+\mathrm{tBHQ}$ & $1.92 \pm 0.17^{\star}$ & $42.33 \pm 4.13^{\star}$ & $3.16 \pm 0.27^{\star}$ \\
\hline
\end{tabular}

\section{Expression of pulmonary Nrf2}

Compared with the sham control group, expression of pulmonary Nrf2 was significantly increased in the I/R group $(\mathrm{P}<0.01)$ and RPC group $(\mathrm{P}<0.01$; Table 2 and Figure 1$)$. Compared with the I/R group, pulmonary expression of Nrf2 was significantly increased in the RPC group $(\mathrm{P}<0.01)$. After tBHQ intervention, Nrf2 expression was significantly increased in pulmonary tissues compared to the vehicle group $(\mathrm{P}<0.01)$. Compared with the RPC group, Nrf2 expression was increased in the RPC+tBHQ group $(\mathrm{P}<0.01)$. Moreover, compared with the RPC group, Nrf2 expression was significantly decreased in pulmonary tissues in the RPC+ATRA group $(\mathrm{P}<0.01)$.

Table 2. mRNA and protein Nfr2 expression in each group.

\begin{tabular}{lcr}
\hline Group & mRNA expression & Protein expression \\
\hline Sham & $1.06 \pm 0.04$ & $0.12 \pm 0.03$ \\
I/R & $2.52 \pm 0.21^{\#}$ & $0.52 \pm 0.07^{\#}$ \\
RPC & $3.81 \pm 0.37^{\# \star}$ & $0.79 \pm 0.15^{\sharp \star}$ \\
I/R+ATRA & $1.95 \pm 0.20^{\star}$ & $0.31 \pm 0.01^{\star}$ \\
RPC+ATRA & $2.47 \pm 0.19^{\star}$ & $0.35 \pm 0.02^{\star}$ \\
I/R+tBHQ & $4.12 \pm 0.46^{\boldsymbol{}}$ & $1.03 \pm 0.20^{\boldsymbol{}}$ \\
RPC+tBHQ & $7.64 \pm 0.69^{\star}$ & $1.50 \pm 0.38^{\star}$ \\
\hline
\end{tabular}

${ }^{\#} \mathrm{P}<0.01$, compared with sham control group; $\cdot \mathrm{P}<0.01$, compared with $\mathrm{I} / \mathrm{R}$ group; $\triangle \mathrm{P}<0.01$, compared with $\mathrm{I} / \mathrm{R}$ group; ${ }^{\circledR} \mathrm{P}<0.01$, compared with RPC group; ${ }^{\nabla} \mathrm{P}<0.01$, compared with $\mathrm{I} / \mathrm{R}$ group; ${ }^{\star} \mathrm{P}<0.01$, compared with $\mathrm{RPC}$ group. 


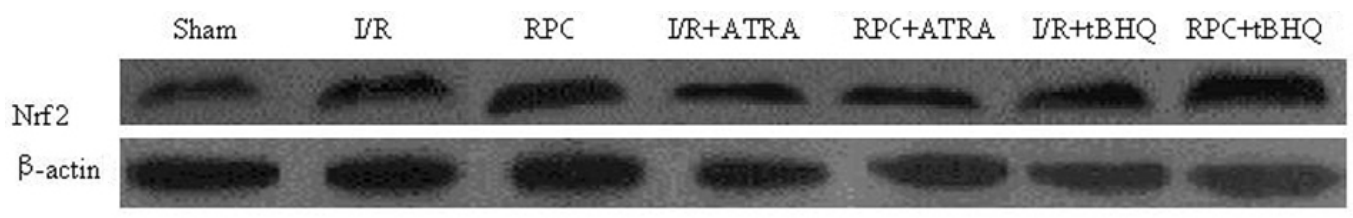

Figure 1. Western blotting analysis in Nrf2 and $\beta$-actin in each group.

\section{Histopathological changes in pulmonary tissues}

In the sham control group, no abnormalities, such as edema and inflammatory cell infiltration, were observed in the alveolar septum of the animals. There was no effusion in the alveolar space. However, histopathological lesions were observed in the I/R+ATRA, I/R, and $\mathrm{I} / \mathrm{R}+\mathrm{tBHQ}$ groups, including interstitial edema, inflammatory cell infiltration, rupture of pulmonary alveoli, partial atrophy, compensatory emphysema, infiltration of red blood cells, inflammatory cells, and effusion. In contrast, in the RPC + ATRA, RPC, and RPC + tBHQ groups, slight edema within pulmonary alveoli was observed, as well as inflammatory cell infiltration and hemorrhage into the alveolar space, which showed remarkable attenuation. In addition, the injury severity of the pulmonary tissues remarkably decreased compared with that in the I/R group, as indicated in Figure 2.

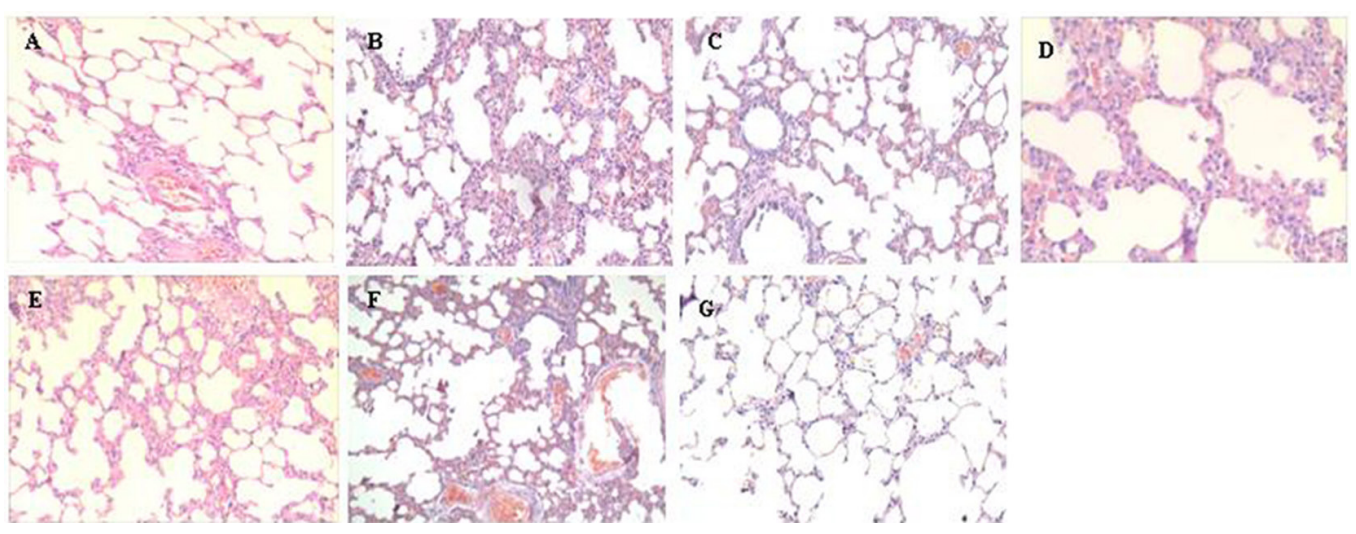

Figure 2. Histopathological changes in the pulmonary tissues of the sham control group (A), I/R group (B), RPC group (C), I/R+ATRA group (D), RPC+ATRA (E), I/R+tBHQ group (F), and RPC +tBHQ group (G) using hematoxylin and eosin staining under a magnification of $100 \mathrm{X}$.

\section{DISCUSSION}

The Nrf2 signal pathway is an important mediator of the defense system in mammals. In addition, it plays important roles in the defense of oxidative stress, anti-apoptosis process, anti-inflammation, regulation of carcinoma, and regulation of immune functions (Sykiotis and Bohmann, 2008; Wu et al., 2011; Al-Huseini et al., 2013). Imbalance in the Nrf2 signal pathway may induce increased sensitivity to various oxidative stresses in the human body, enhance the toxic effects of oxidative stress, and result in cellular function disorder, apoptosis, and even necrosis (Hur and Gray, 2011). 
The Nrf2 signal pathway mainly consists of Nrf2, Kelch-like ECH-associated protein (Keap1), and antioxidant response element. In unstimulated cells, Keap1 binds with and brings Nrf2 into a culin3-dependent E3 complex that ubiquitinates Nrf2, resulting in rapid turnover of the protein (He and Ma, 2010). In addition, Keap1 may induce Nrf2 ubiquitination, leading to the degradation of Nrf2 in the presence of protease. During oxidative stress, oxidation of the hydrosulfide group on Keap1 or the phosphorylation of serine and/or threonine on Nrf2 may result in dissociation of the Keap1-Nrf2 complex, after which Nrf2 is transferred from the cytoplasm to the nucleus (Hochmuth et al., 2011; Taguchi et al., 2011). Subsequently, the Nrf2 in the nucleus can bind to antioxidant response elements by forming a dipolymer with Maf using the bZIP structure, which triggers activation of antioxidant response element and activates the expression of various proteases (e.g., g-glutamyl cysteine synthetase, NAD(P)H quinone oxidoreductase 1, and heme oxygenase-1) and gene transcription of anti-oxidant proteins (Osburn and Kensler 2008). It has been well established that Nrf2 plays a pivotal role in pulmonary disorder and injury. In a previous study, pulmonary injury induced by multiple oxidative damages and other inflammatory factors was observed in the absence of Nrf2. In a study aimed to clarify the protective role of $\mathrm{Nrf} 2$ in the development of emphysema, the susceptibility of Nrf2-knockout mice exposed to cigarette smoke to emphysema was investigated. The results indicated that neutrophilic lung inflammation and permeability in lung damage significantly increased in Nrf2 knockout mice; a significant increase was observed in pulmonary elastase activity, macrophage, and production of oxidative stress in bronchoalveolar lavage fluid (Iizuka et al., 2005). Cho et al. (2002) reported that Nrf2 played a significant protective role against pulmonary hyperoxic injury in mice. Furthermore, Nrf2-dependent antioxidants and DJ-1 (a protein that stabilizes Nrf2 by impairing Keap1-dependent proteasomal degradation of Nrf2) expression was negatively associated with the severity of chronic obstructive pulmonary disease (Malhotra et al., 2008).

In our study, an antagonist (ATRA) and agonist (tBHQ) of the Nrf2 signaling pathway were used to investigate the role of this pathway in regulating $\mathrm{I} / \mathrm{R}$ injury. Our results indicated that the expression of Nrf2 increased in rats with I/R injury after remote postconditioning. In addition, the severity of injury was reduced after treatment with RPC. After intervention with ATRA, Nrf2 expression was significantly decreased, combined with a decreased in I/R injury severity. However, after intervention with tBHQ, a remarkable decrease in Nrf2 as well as deterioration of I/R injury was observed, and no significant protective effects were observed in the rats with I/R injury after RPC. Intervention of Nrf2 may induce a significant increase in Nrf2 expression, resulting in remarkable alleviation of I/R injury. Remarkable protective effects were observed after RPC. This indicates that Nrf2 plays an important role in the endogenous protection of $\mathrm{I} / \mathrm{R}$ injury.

In conclusion, our results indicate that $\mathrm{Nrf} 2$ is involved in the protective effects of $\mathrm{RPC}$ in rats with I/R injury. Activation of Nrf2 was associated with the alleviation of pulmonary injury, while deactivation of Nrf2 was related to deterioration of the injury.

\section{Conflicts of interest}

The authors declare no conflict of interest.

\section{REFERENCES}

Al-Huseini LM, Aw Yeang HX, Sethu S, Alhumeed N, et al. (2013). Nuclear factor-erythroid 2 (NF-E2) p45-related 
factor-2 (Nrf2) modulates dendritic cell immune function through regulation of p38MAPK-CREB/ATF1 signalling. J. Biol. Chem. 288: 22281-22288.

Beillard E, Pallisgaard N, van der Velden VH, Bi W, et al. (2003). Evaluation of candidate control genes for diagnosis and residual disease detection in leukemic patients using 'real-time' quantitative reverse-transcriptase polymerase chain reaction (RQ-PCR) - a Europe against cancer program. Leukemia 17: 2474-2486.

Cho HY, Jedlicka AE, Reddy SP, Kensler TW, et al. (2002). Role of NRF2 in protection against hyperoxic lung injury in mice. Am. J. Respir. Cell. Mol. Biol. 26: 175-182.

He X and Ma Q (2010). Critical cysteine residues of Kelch-like ECH-associated protein 1 in arsenic sensing and suppression of nuclear factor erythroid 2-related factor 2. J. Pharmacol. Exp. Ther. 332: 66-75.

Hochmuth CE, Biteau B, Bohmann D and Jasper H (2011). Redox regulation by Keap1 and Nrf2 controls intestinal stem cell proliferation in Drosophila. Cell Stem Cell 8: 188-199.

Hur W and Gray NS (2011). Small molecule modulators of antioxidant response pathway. Curr. Opin. Chem. Biol. 15: $162-173$.

Iizuka T, Ishii Y, Itoh K, Kiwamoto T, et al. (2005). Nrf2-deficient mice are highly susceptible to cigarette smoke-induced emphysema. Genes Cells 10: 1113-1125.

Ke JJ, Yu FX, Rao Y and Wang YL (2010). Adenosine postconditioning protects against myocardial ischemia-reperfusion injury though modulate production of TNF-a and prevents activation of transcription factor NF-kappaB. Mol. Biol. Rep. 38: 531-538.

Malhotra D, Thimmulappa R, Navas-Acien A, Sandford A, et al. (2008). Decline in NRF2-regulated antioxidants in chronic obstructive pulmonary disease lungs due to loss of its positive regulator, DJ-1. Am. J. Respir. Crit. Care Med. 178: 592-604.

Osburn WO and Kensler TW (2008). Nrf2 signaling: an adaptive response pathway for protection against environmental toxic insults. Mutat. Res. 659: 31-39.

Satoh K (1978). Serum lipid peroxide in cerebrovascular disorders determined by a new colorimetric method. Clin. Chim. Acta 90: 37-43.

Sykiotis GP and Bohmann D (2008). Keap1/Nrf2 signaling regulates oxidative stress tolerance and lifespan in Drosophila. Dev. Cell 14: 76-85.

Taguchi K, Motohashi H and Yamamoto M (2011). Molecular mechanisms of the Keap1-Nrf2 pathway in stress response and cancer evolution. Genes Cells 16: 123-140.

Tang Y, Mennander A, Oksala N, Atalay M, et al. (2010). Postconditioning and remote postconditioning of ischemic rat cardiac grafts. Eur. Surg. Res. 45: 1-8.

Tsubota H, Marui A, Esaki J, Bir SC, et al. (2010). Remote postconditioning may attenuate ischaemia-reperfusion injury in the murine hindlimb through adenosine receptor activation. Eur. J. Vasc. Endovasc. Surg. 40: 804-809.

Unver A, Felek S, Paddock CD, Zhi N, et al. (2001). Western blot analysis of sera reactive to human monocytic ehrlichiosis and human granulocytic ehrlichiosis agents. J. Clin. Microbiol. 39: 3982-3986.

Wu TY, Khor TO, Saw CL, Loh SC, et al. (2011). Anti-inflammatory/anti-oxidative stress activities and differential regulation of Nrf2-mediated genes by non-polar fractions of tea Chrysanthemum zawadskii and licorice Glycyrrhiza uralensis. AAPS J. 13: 1-13.

Xin P, Zhu W, Li J, Ma S, et al. (2010). Combined local ischemic postconditioning and remote preconditioning recapitulate cardioprotective effects of local ischemic preconditioning. Am. J. Physiol. Heart Circ. Physiol. 298: H1819-H831. 